\title{
PERCEPCIONES EN EL USO DE LAS REDES SOCIALES Y SU APLICACIÓN EN LA ENSEÑANZA DE LAS MATEMÁTICAS
}

\section{PERCEPTIONS ON THE USE OF SOCIAL NETWORKS AND THEIR APPLICATION IN THE TEACHING OF MATHEMATICS}

\author{
Dra. Ivanovnna Milqueya Cruz Pichardo \\ ivanovnnacruz@pucmm.edu.do
}

Pontificia Universidad Católica Madre y Maestra. Departamento de Ciencias Básicas. c/ Julia G. Madsen esq. Ave. Sarasota. Plaza Universitaria. Edif. B1, sexta planta, Santo Domingo, (República Dominicana)

El uso de las redes sociales han tomado un papel importante en los procesos de enseñanza aprendizaje, debido a las diferentes ventajas que ellas aportan en cuanto a la comunicación y trabajo colaborativo, permitiendo un proceso compartido de conocimiento que favorece la socialización del grupo y la interacción continua de todos los actores involucrados. Nuestro trabajo se enmarca dentro de una investigación más amplia que perseguía analizar la implementación del uso de las redes sociales en la resolución de problemas matemáticos en estudiantes no universitarios. El estudio nos permitió observar la percepción positiva que poseen los alumnos hacia el uso de las redes sociales para la enseñanza de las Matemáticas. Palabras Clave: Redes sociales, resolución de problemas, enseñanza de las Matemáticas.

The use of social networks have taken an important role in teaching and learning processes, due to the various benefits they bring in terms of communication and collaborative work, allowing sharing of knowledge process that favors the socialization of group interaction continuous all stakeholders. Our work is part of a broader investigation that analyzes the implementation of the use of social networks in solving mathematical problems for not college students. The study allowed us to observe the positive perception that students have towards the use of social networks for teaching mathematics.

Keywords: Social networking, problem solving, mathematics teaching.

Píxel-Bit. Revista de Medios y Educación. $N^{o} 48$ Enero 2016. ISSN: 1133-8482. e-ISSN: 2171-7966. doi: http://dx.doi.org/10.12795/pixelbit.2016.i48.11 


\section{Introducción.}

Las Redes sociales (RS) son un fenómeno característico de nuestro tiempo y que progresivamente van ampliando su penetración al terreno educativo como han puesto de manifiesto diferentes Informes (García et al., 2010; Durall, Gros, Maina, Johnson \& Adams, 2012). Su significación ha ido ampliándose tanto por los que los utilizan para el trabajo colaborativo, como por aquellos que empiezan a considerarlas como posibles sustitutas de las plataformas de teleformación (Arnold \& Trena, 2010; Túñez $\&$ Sixto, 2012).

En su definición nos encontramos con diferentes perspectivas; las que se centran en la comunicación y la colaboración como variables fundamentales; y las que se basan en el estudio de las características estructurales de sus herramientas (Cabero, Barroso, Llorente \& Marín, 2013). Por aportar alguna definición citar la De Haro (2010) que las define como una estructura social que se puede representar mediante nodos conectados por aristas, donde los nodos representan a los individuos y las aristas las relaciones entre ellos. Por su parte Boyd y Ellison (2007), consideran que son herramientas que permiten a los individuos construir un pérfil público o semipúblico dentro de un sistema delimitado, que les consiente articular una lista de diferentes usuarios con los que comparte una conexión y además poder ver las conexiones que suceden dentro de su sistema. Por último señalar que para Cabero (2012) son un multientorno que permiten la discusión de diferentes tipos de problemas en espacios interactivos y flexible de aprendizaje.

Respecto a su tipología, nos encontramos con diferentes tipos: verticales-horizontales, profesionales-de ocio, abiertas-cerradas, gratuitas-de pago, libres-alojadas en servidores propios,... (De Alsola, 2009; De Haro, 2011), que implican posibilidades diferentes a las de su puesta en acción.

En relación a sus posibilidades y de acuerdo con la propuesta formulada por diferentes autores (Cachia, 2008; Ortega \& Gacitúa, 2008; Santamaría, 2008; Camacho, 2010; De Haro, 2010; Romero, 2011; Joosten, 2012; Cabero \& Marín, 2013; Rodríguez, 2013) podemos señalar las siguientes: permiten la creación natural de una inteligencia colectiva, amplian las fronteras del proceso de enseñanza aprendizaje, facilitan la comunicación, favorecen la construcción colaborativa del conocimiento, implican un cambio en la gestión de los procesos de enseñanza, fomentan una visión compartida del conocimiento, favorecen la socialización del grupo, son espacio de encuentro entre los diferentes actores del proceso enseñanzaaprendizaje; permiten centralizar en un único sitio todas las actividades docentes, aumentan la fluidez y sencillez de la comunicación entre profesores y alumnos, facilitan la coordinación y trabajo de diversos grupos de aprendizaje, aumentan la interacción entre profesores y alumnos, facilitan la retroalimentación de los estudiantes a tiempo, mejora el aprendizaje, favorece la creación de un aprendizaje activo, permiten recrear grupos de trabajo y de actividades socializadoras, son fáciles de incorporar a la enseñanza ya que los alumnos se encuentran familiarizado con ellas, y se genera en el estudiante un proceso de análisis y síntesis reforzando su actitud crí-tica al estar o no estar de acuerdo con otras opiniones disponibles en la herramienta.

Y por lo que se refiere a sus inconvenientes podemos señalar las siguientes: tener 
precaución con la privacidad, su incorporación exige del profesorado crear actividades motivadoras para que no se conviertan en un distractor y elemento lúdico y de ocio, la falta de formación de los padres en estos aspectos podría traer desconfianza y poco control al momento de usarlas en casa, y el riesgo del acoso cibernético (Roig, 2011; Aydin, 2012; Osborne, 2012).

Su utilización implica para el docente el desempeño de nuevos roles, que superan la mera transmisión de contenidos. Llorente (2012), habla que el docente debe asumir dentro de este proceso los papeles de guía, dinamizador y orientador. A ellos, incorporaríamos el diseñar las tareas que permitan el desarrollo de competencias de selección y procesamiento de información. Por su parte Minocha, Schroeder y Schneider (2011) señalan cuatro roles fundamentales que el docente debe desempeñar en las mismas: pedagógico (facilita el aprendizaje a través de actividades interactivas, la reflexión crítica y la retroalimentación en línea), social (facilita la creación y el crecimiento de una comunidad estudiantil en línea a través de una comunicación frecuente y positiva), de dirección (guía en el procedimiento, en la organización y la administración de las tareas a ejecutar), y técnica (proporciona apoyo técnico). Aunque también en estas funciones se encuentran la de seleccionar las RS a utilizar, para lo cual puede movilizar diferentes criterios que permitan lograr aprendizajes significativos en los estudiante, que sea efeciente en su uso, acceso y costo, y que esté basada en los principios del trabajo colaborativo para su mayor rendimiento como recurso educativo y que permita incorporar la enorme cantidad de elementos multimedia que tenemos a disposición para lograr nuestros fines educativos (Joosten, 2012; Llorente, 2012).

Para nuestro estudio, después de analizar los factores y aspectos que necesitábamos en la red, establecimos que la plataforma más apropiada era Edmodo, ya que es un servicio cerrado, nos permite en envío de mensajes de texto, adjuntar archivos, corregir trabajos. Nos permite trabajar con alumnos de todas las edades inclusive niños menores de 14 años ya que tenemos acceso a supervisión directa de los padres. Además podemos tener contacto continuo con los alumnos lo que nos permite un apoyo sistemático de las clases presenciales (De Haro, 2012).

Por lo que se refiere a las estrategias grupales que se pueden utilizar en las RS, señalar que son diversas: método de la solución de problemas, método de proyectos individuales, método del caso, método del contrato, investigación grupal, observación tríadica, seminario, coloquio, simulación didáctica y ponencia científica (Chávez, Solis \& Iriarte, 2012).

Para finalizar estos comentarios, señalar dos aspectos: uno, que cuando se han llevado a cabo investigaciones que han analizado el nivel de satisfacción que los alumnos tienen al participar en experiencias formativas con ellas, los resultados han sido bastante positivas (Irwin, Ball, Desbrow \& Leveritt, 2012; Cabero \& Marín, 2013), y dos, que sobre ellas se han llevado a cabo más estudios y reflexiones teóricas que investigaciones (Veletsianos \& Navarrete, 2012; López, Vásquez \& Sarasola, 2015), y estas además son muy escasas en el terreno de las matemáticas, que es el ámbito en el cual se desarrolla nuestra experiencia. 


\section{Metodología.}

\subsection{Objetivos y problemas de investigación.}

El presente trabajo se enmarca dentro de una investigación más amplia que perseguía los siguientes objetivos: a) Diseñar una red social cerrada centrada en la resolución de problemas matemáticos, b) Determinar el rendimiento académico alcanzado por los estudiantes en el proceso de implementación de la acción formativa basada en las redes sociales y la resolución de problemas, c) Estudiar la percepción que poseen los estudiantes no universitarios respecto a la utilidad de las redes sociales, d) Conocer la preferencia que poseen los estudiantes no universitarios en cuanto al trabajo en grupo, e) Analizar la actitud hacia las matemáticas de los estudiantes no universitarios, y f) Comprobar la eficacia de las Redes Sociales como instrumento facilitador de las interacciones sociales en los alumnos no universitarios.

En el trabajo actual nos centraremos específicamente en el objetivo «c» citado, intentando también conocer si la experiencia realizada de la utilización de las RS para la enseñanza de la resolución de problemas matemáticos había modificado las percepciones iniciales que tenían hacia las mismas. Analizando también las posibles influencias que la variable género tenía sobre las mismas. Las fases que seguimos en el estudio fueron las siguientes:

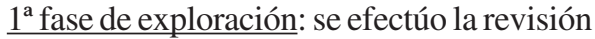
bibliográfica y se identificó el centro donde realizamos el estudio.

$\underline{2^{a} \text { fase de diseño: }}$ se centró en la selección, diseño y construcción de los contenidos y materiales a utilizarse en la parte experimental.
Además de la construcción y fiabilización de los instrumentos que se utilizarían en el estudio. También se evaluaron diferentes RS, y se seleccionó la utilizada en nuestro estudio, al mismo tiempo se procedió a preparar la plataforma EDMODO, creamos los grupos, establecimos los permisos.

$3^{\mathrm{a}}$ fase de aplicación: Se desarrolló la experiencia, y se aplicaron los instrumentos de recogida de información.

$4^{\mathrm{a}}$ fase de recogida y análisis de datos: análisis de datos y obtención de conclusiones.

\subsection{La población y muestra del estudio.}

La experiencia se realizó durante el curso académico 2013-2014, en un centro privado de clase media-alta que se encuentra localizado en una zona céntrica de la ciudad de Santo Domingo, en la RD. La investigación se desarrolló en el décimo grado, en la asignatura de Geometry que pertenece al programa de inglés de este grado, y fue cursada por 50 estudiantes, con edades comprendidas entre los 14 a 17 años. Siendo la mayoría de $15(\mathrm{f}=13,26,0 \%)$ y 16 años $(\mathrm{f}=32$, $64,0 \%)$. El 58,0\% ( $\mathrm{f}=29)$ eran mujeres y el $42,0 \%(\mathrm{f}=21)$ eran hombres.

Los contenidos impartidos se agrupaban en los siguientes grandes bloques: Explorando la Geometría; Razonamiento en Geometría; y Relación de ángulos, líneas y construcciones.

\subsection{Cuestionario de la percepción hacia las redes sociales.}

Para analizar las percepciones que los alumnos tenían hacia las RS, construimos un cuestionario «ad hoc», que estaba conformado por cuatro grandes dimensiones; 
tres referidas directamente a las RS y una a su aplicación en el terreno de la resolución de problemas matemáticos.

1) Aspectos relacionados con el alta en las redes sociales: perseguía obtener información sobre las RS que los encuestados conocían, identificar si los estudiantes tenían cuenta en una de esas redes y si eran cuentas activas que la utilizaban por lo menos una vez al día. También se perseguía conocer el tiempo que llevan los encuestados con una cuenta activa en las redes sociales.

2) Aspectos relacionados con el uso de las redes sociales: con ella pretendíamos conocer qué actividades realizan los estudiantes dentro de las redes sociales.

3) Aspectos relacionados con las herramientas de las redes sociales: pretendíamos recoger información sobre las herramientas más usadas por los estudiantes y que actividades desarrollaban en su tiempo en la red.

4) Aspectos relacionados con la matemática y la resolución de problemas: Se refiere específicamente a la propuesta en análisis, a fin de indagar si los estudiantes entendían apropiado el uso de las redes sociales para la resolución de problemas y para trabajar matemáticas en ella.

A estas dimensiones le incorporamos una serie de preguntas destinadas a conocer algunas características biográficas del encuestado: género, edad y grado que estaban cursando.

Para su construcción tuvimos en cuenta las sugerencias de McMillan y Schumacher (2005) y las características, que según Sampieri, Fernández y Baptista (2013), deben tenerse presente al momento de elaborar las preguntas de un cuestionario: ser claras, precisas y de fácil comprensión para los sujestos encuestados; elaborarlas lo más breve posible, ya que las preguntas largas pueden ocasionar distracción y perdida de motivación en los encuestados; tener un vocabulario simple, directo y familiar para los participantes; deben referirse a un solo aspecto o una relación lógica; no deben inducir la respuesta; evitar preguntas que nienguen el asunto que se interroga; y no deben ofender al encuestado.

La primera versión del instrumento estubo constituida por 26 items de diferentes tipologías: Preguntas cerradas dicótomicas, preguntas cerradas excluyentes, preguntas cerradas no excluyentes, las cuales permitían seleccionar diversas opciones dentro de la misma pregunta y tenían la opción «otros» que nos permitía ampliar la lista de redes sociales. Además se incorporaron un número de preguntas con construcción tipo Likert, para conocer el grado de acuerdo o desacuerdo del estudiante con el reactivo propuesto.

Para su validación decidimos hacer una prueba piloto donde seleccionamos una muestra de 81 jóvenes entre las edades de 13-18 años de diferentes grados y en un centro educativo que tuviese las mismas características que la muestra donde posteriormente realizamos la investigación. Utilizando para ello el índice de fiabilidad de Alfa Crombach en las preguntas que lo permitían, obteniéndose un índice del 0.802 , lo que nos permite considerarlo fiable según lo planteado por Barroso y Cabero (2010). Para afianzar su fiabilidad obtuvimos la correlación Item-Total (Tabla 1), valores que nos indicaban la necesidad de no tener que eliminar ningún ítem, pues ello no repercutía en el aumento del índice de fiabilidad del instrumento. 


\begin{tabular}{|c|c|c|c|c|}
\hline & $\begin{array}{c}\text { Media de la escala si } \\
\text { se elimina el } \\
\text { elemento }\end{array}$ & $\begin{array}{l}\text { Varianza de la escala si } \\
\text { se elimina el elemento }\end{array}$ & $\begin{array}{l}\text { Correlación } \\
\text { elemento- total } \\
\text { corregido }\end{array}$ & $\begin{array}{c}\text { Alfa de Crombach si } \\
\text { se elimina el elemento }\end{array}$ \\
\hline V1 & 44.8889 & 35.540 & .482 & .786 \\
\hline V2 & 45.0370 & 36.632 & .413 & .791 \\
\hline $\mathbf{V 3}$ & 45.0556 & 35.941 & .549 & .782 \\
\hline V4 & 44.7778 & 35.988 & .585 & .780 \\
\hline V5 & 44.6852 & 39.347 & .186 & .806 \\
\hline V6 & 44.3333 & 36.522 & .555 & .783 \\
\hline V7 & 44.5556 & 37.553 & .341 & .796 \\
\hline v8 & 46.0926 & 40.743 & .055 & .813 \\
\hline V9 & 44.6667 & 36.783 & .438 & .789 \\
\hline V10 & 44.9444 & 36.239 & .568 & .781 \\
\hline V11 & 45.1667 & 39.531 & .174 & .806 \\
\hline V12 & 46.0556 & 40.003 & .139 & .808 \\
\hline V13 & 44.9259 & 36.218 & .666 & .778 \\
\hline V14 & 44.7407 & 34.665 & .717 & .770 \\
\hline V15 & 44.5741 & 36.209 & .469 & .787 \\
\hline V16 & 44.6111 & 39.519 & .171 & .807 \\
\hline V17 & 44.7407 & 39.547 & .227 & .802 \\
\hline
\end{tabular}

Tabla 1. Correlación Ítem-Total Cuestionario de la Percepción hacia las Redes Sociales.

Otro aspecto que tuvimos en cuenta, en la prueba piloto, fue las respuestas que obtuvimos en las preguntas 4-6, específicamente en la opción «Otros». Utilizamos las respuestas para verificar si el listado de opciones eran apropiadas o no, si debíamos eliminar una opción o agregar otras. Los resultados encontrados nos llevaron a tomar la decisión de mantener en la lista las opciones que eran conocidos por más del $25 \%$ de la población, para evitar tener una lista muy grande de opciones, y darle seguimiento en la aplicación del instrumento a las que tenían cantidad menor que el $20 \%$. Agregamos a nuestro instrumento Instagram y Pinterest ya que ambas eran conocidas por 


\begin{tabular}{|c|c|c|c|c|c|c|}
\hline \multirow[t]{2}{*}{ Redes } & \multicolumn{2}{|c|}{$\begin{array}{l}\text { ¿Cuál de las siguientes } \\
\text { redes sociales conoces? }\end{array}$} & \multicolumn{2}{|c|}{$\begin{array}{l}\text { ¿En cuál de las siguientes } \\
\text { redes tienes una cuenta? }\end{array}$} & \multicolumn{2}{|c|}{$\begin{array}{l}\text { ¿En cuál tienes una } \\
\text { cuenta activa? }\end{array}$} \\
\hline & Pre Exp & Post Exp & Pre Exp & Post Exp & Pre Exp & Post Exp \\
\hline & $f(\%)$ & $f(\%)$ & $f(\%)$ & $\mathrm{f}(\%)$ & $\mathrm{f}(\%)$ & $f(\%)$ \\
\hline Facebook & 47 (94) & $50(100)$ & 47 (94) & $50(100)$ & $43(86)$ & $45(90)$ \\
\hline Twitter & $46(92)$ & $48(96)$ & $43(86)$ & $48(96)$ & $32(64)$ & $32(64)$ \\
\hline Myspace & $32(64)$ & $32(64)$ & $6(12)$ & $6(12)$ & $0(0)$ & $0(0)$ \\
\hline Linkedin & $8(16)$ & $8(16)$ & $2(4)$ & $2(4)$ & $0(0)$ & $0(0)$ \\
\hline Skype & 47 (94) & 47 (94) & $40(80)$ & 47 (94) & $22(44)$ & $24(48)$ \\
\hline Youtube & 47 (94) & 47 (94) & 37 (74) & 47 (94) & $27(54)$ & $30(60)$ \\
\hline Instagram & $48(96)$ & $48(96)$ & $32(64)$ & $48(96)$ & $26(56)$ & $30(60)$ \\
\hline Pinterest & $19(38)$ & $24(48)$ & $10(20)$ & $24(48)$ & 7 (14) & $12(24)$ \\
\hline Tumblr & $3(6)$ & $4(8)$ & $3(6)$ & $4(8)$ & $1(2)$ & $1(2)$ \\
\hline Line & $1(2)$ & $6(12)$ & $1(2)$ & $6(12)$ & $0(0)$ & $0(0)$ \\
\hline Outlook & $1(2)$ & $1(2)$ & $0(0)$ & $0(0)$ & $0(0)$ & $0(0)$ \\
\hline Snapshot & $1(2)$ & $10(20)$ & $0(0)$ & $10(20)$ & $1(2)$ & $4(8)$ \\
\hline Vine & $2(4)$ & $6(12)$ & $1(2)$ & $6(12)$ & $1(2)$ & $1(2)$ \\
\hline Path & $1(2)$ & $1(2)$ & $0(0)$ & $0(0)$ & $0(0)$ & $0(0)$ \\
\hline Whatsapp & $2(4)$ & $36(72)$ & $2(4)$ & $36(72)$ & $2(4)$ & $9(18)$ \\
\hline BBM & $1(2)$ & $1(2)$ & $1(2)$ & $1(2)$ & $0(0)$ & $0(0)$ \\
\hline Edmodo & $0(0)$ & $50(100)$ & $0(0)$ & $50(100)$ & $0(0)$ & $50(100)$ \\
\hline Google + & $0(0)$ & $15(30)$ & $0(0)$ & $15(30)$ & $0(0)$ & $0(0)$ \\
\hline Hi5 & $1(2)$ & $1(2)$ & $1(2)$ & $1(2)$ & $0(0)$ & $0(0)$ \\
\hline Ninguna & $0(0)$ & $0(0)$ & $0(0)$ & $0(0)$ & $0(0)$ & $0(0)$ \\
\hline
\end{tabular}

Tabla 2. Resultado de la pregunta de las redes sociales que conocen.

Píxel-Bit. Revista de Medios y Educación. No 48 Enero 2016. ISSN: 1133-8482. e-ISSN: 2171-7966. doi: http://dx.doi.org/10.12795/pixelbit.2016.i48.11 


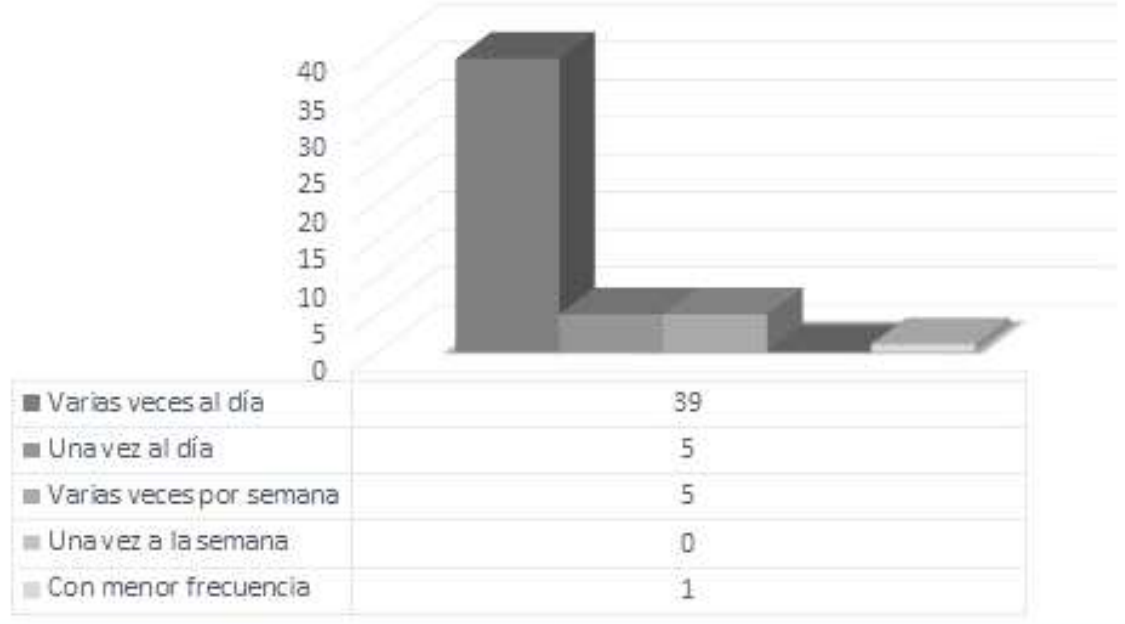

Figura 1. Resultado de la pregunta ¿Cuántas veces usas tu red social?.

un $37 \%$ y un $31 \%$ respectivamente y decidimos eliminar la opción LinkedIn ya que solo el $12 \%$ de los encuestados la conocían. Quedando la lista final constituida por: Facebook, Twitter, Myspace, Skype, YouTube, Instagram, Pinterest, Otras, y Ninguna. En el anexo del presente artículo puede observarse la versión final del instrumento.

Señalar que en nuestra investigación el instrumento se administró dos veces, antes y después de realizar la experiencia formativa, con el objeto de conocer si ella influía en la modificación de las percepciones y los usos que inicialmente tenían los estudiantes respecto a las RS.

\section{Resultados alcanzados.}

A continuación vamos a presentar los resultados obtenidos, tanto antes de comenzar la experiencia como al finalizar la misma. Y los iremos presentando de acuerdo a las diferentes dimensiones que conformaban el cuestionario.

En relación con los aspectos relacionados con las redes sociales, en la Tabla 2 presentamos los resultados alcanzados respecto a las redes que conocían.

Como podemos observar las redes más conocidas por los estudiantes entrevistados son el Facebook, Twitter, Skype, Instagram y Youtube tanto antes como después de la experiencia. Y por lo que se refiere a las que desconocían se destacaban dos: Edmodo y Google + . Recordemos que con la primera fue con la que se realizó nuestro estudio.

La RS donde los estudiantes tienen más cuentas creadas es en Facebook, tanto antes como después de participar en la experiencia, seguida por Twitter e Instagram. Por supuesto que después de la experiencia podemos darnos cuenta que en Edmodo tiene un 100 $\%$ ya que se utilizó como plataforma base para el desarrollo de nuestro estudio.

Por lo que se refiere a las cuentas activas, lo primero a señalar es que no en todos los 


\begin{tabular}{|l|c|c|c|c|c|}
\hline \multicolumn{1}{|c|}{ Utilizo las redes sociales para: } & \multicolumn{2}{|c|}{$\begin{array}{c}\text { Pre Experiencia } \\
\text { Formativa }\end{array}$} & \multicolumn{2}{|c|}{$\begin{array}{c}\text { Post Experiencia } \\
\text { Formativa }\end{array}$} & Diferencia \\
\cline { 2 - 5 } & Media & $\begin{array}{c}\text { Desv. } \\
\text { Standard }\end{array}$ & Media & $\begin{array}{c}\text { Desv. } \\
\text { Standard }\end{array}$ & \\
\hline Conocer gente nueva & 2.46 & 1.092 & 2.84 & 1.017 & +0.38 \\
\hline Porque todos mis amigos están & 3.14 & .808 & 3.22 & .737 & +0.08 \\
\hline Para comentar & 2.50 & 1.035 & 2.76 & .938 & +0.26 \\
\hline Porque es divertida & 3.24 & .716 & 3.34 & .557 & +0.1 \\
\hline $\begin{array}{l}\text { Para saber de gente que hace tiempo } \\
\text { no tengo relación }\end{array}$ & 2.98 & 1.040 & 2.98 & 1.040 & 0 \\
\hline $\begin{array}{l}\text { Para mantener el contacto con mis } \\
\text { amigos }\end{array}$ & 3.52 & .707 & 3.60 & .606 & +0.08 \\
\hline $\begin{array}{l}\text { Para hacer tareas y/o actividades del } \\
\text { colegio }\end{array}$ & 3.10 & .886 & 3.30 & .647 & +0.2 \\
\hline
\end{tabular}

Tabla 3. Medias, Desviación y diferencia sobre pretest y el potest de la respuesta para que utilizó las redes sociales.

casos tenían los alumnos una cuenta activa. Además podemos observar que solo 3 personas poseen una solo cuenta activa lo que equivale al $6 \%$ de la población encuestada, y que esas cuentas están activa en Facebook o Instagram. También podemos ver que la mayoría tiene una red donde pueden postear fotos y ver videos.

Además de conocer en que redes tenían cuentas activas, queríamos saber desde cuándo la tenían activa, encontrándonos que el $12 \%(\mathrm{f}=6)$ la tenía de hace más de 6 años, mientras que el $48 \%$ ( $\mathrm{f}=24)$ la tenía entre 4 y 5 años, y solo el $4 \%$ ( $\mathrm{f}=2$ ) tiene menos de 6 meses con una cuenta activa. Dato que confirmaba que las RS es una tecnología usual para los estudiantes.

Por lo que se refiere a en cuántas redes habian introducido su perfil, nos encontramos que el $32 \%$ ( $\mathrm{f}=16$ ) lo habían realizado en varias, y solo el $10 \%$ ( $\mathrm{f}=5$ ) han introducido su perfil en una sola cuenta.
Y por lo que respecta a la frecuencia con que visitaban sus redes sociales, el $78 \%$ ( $\mathrm{f}=39$ ) nos informó que entran en redes sociales varias veces al día y solo un alumno señaló que entraba con una frecuencia menor a una vez por semana (Figura 1).

Por lo que se refiere al uso de las redes sociales que hacían nuestros estudiantes. En la Tabla 3, presentamos los resultados alcanzados, de nuevo presentamos los resultados antes y después de participar en la experiencia. Para su correcta interpretación, téngase en cuenta que el 1 era igual a «totalmente en desacuerdo» $y$ «4» «totalmente de acuerdo».

Como podemos observar los alumnos suelen estar «de acuerdo» con las diferentes opciones por las que se les preguntaba, y en todos los casos se supera la puntuación media de 2. Sobresalen las opciones de respuestas destinadas a «mantener el 


\begin{tabular}{|c|c|c|c|c|c|}
\hline \multirow[t]{2}{*}{$\begin{array}{l}\text { Utilizo mi tiempo en las redes } \\
\text { sociales para: }\end{array}$} & \multicolumn{2}{|c|}{$\begin{array}{l}\text { Pre Experiencia } \\
\text { Formativa }\end{array}$} & \multicolumn{2}{|c|}{$\begin{array}{l}\text { Post Experiencia } \\
\text { Formativa }\end{array}$} & \multirow[t]{2}{*}{ Diferencia } \\
\hline & Media & Desv. St. & Media & Desv. St. & \\
\hline Jugar en la red & 1.86 & .904 & 1.86 & .904 & 0 \\
\hline Para murmurar & 3.00 & .833 & 3.00 & .833 & 0 \\
\hline $\begin{array}{l}\text { Informarme sobre las cosas que } \\
\text { me interesan }\end{array}$ & 2.54 & .952 & 2.90 & .909 & +0.36 \\
\hline Actualizar mi perfil & 3.00 & .926 & 3.08 & .922 & +0.08 \\
\hline Mandar mensajes privados & 1.54 & .838 & 1.92 & 1.085 & +0.38 \\
\hline $\begin{array}{l}\text { Para mantener el contacto con } \\
\text { mis amigos }\end{array}$ & 2.58 & .971 & 2.72 & .970 & +0.14 \\
\hline Compartir o subir fotos & 2.74 & 1.046 & 2.96 & .968 & +0.22 \\
\hline $\begin{array}{l}\text { Hacer tareas y/o actividades del } \\
\text { colegio }\end{array}$ & 3.10 & .886 & 3.30 & .647 & +0.2 \\
\hline
\end{tabular}

Tabla 4. Uso de las redes sociales.

contacto con mis amigos» $\mathrm{y}$ «hacer tareas y/ o actividades del colegio».

Por lo que se refiere a los aspectos relacionados con las herramientas de las redes sociales que solían fundamentalmente utilizar en la Tabla 4, presentamos los resultados alcanzados.

Como podemos observar la mayoría de los alumnos nos informan que suelen utilizar las RS para realizar actividades de carácter social, como son las de mantenerse en contacto con sus amigos (2.58), compartir o subir fotos (2.74) y para murmurar (3.0) sobre aspectos específicos. Aunque también se muestran de acuerdo con su utilización para actividades relacionadas con la formación: informarse sobre las cosas que le interesaban y hacer actividades relacionadas con los estudios (3.10). Nos hemos encontrado también que los alumnos indicaban que no estaban de acuerdo con indicarles que utilizaban la RS para «jugar» (1.86).

Nuestra última dimensión se refería al análisis de los aspectos relacionados con la matemática y resolución de problemas, en la Tabla 5 se presentan los resultados alcanzados.

Es importante resaltar que con la afirmación Me gustaría utilizar las redes sociales para las clases de matemática antes de la experiencia formativa $64 \%(\mathrm{f}=32)$ estaban en desacuerdo con la afirmación, porcentaje que bajó al $60 \%$ ( $\mathrm{f}=30)$ una vez finalizada la experiencia.

Con el objeto de conocer si la participación en la experiencia había tenido consecuencias significativas desde un punto de vista estadístico, formulamos las siguientes hipótesis:

H0 (Hipótesis Nula): No hay diferencias significativas en el uso que realizan de las RS 


\begin{tabular}{|c|c|c|c|c|c|}
\hline \multirow{2}{*}{$\begin{array}{c}\text { Aspectos relacionados con } \\
\text { Matemática y resolución de } \\
\text { problemas }\end{array}$} & \multicolumn{2}{|c|}{$\begin{array}{l}\text { Pre Experiencia } \\
\text { Formativa }\end{array}$} & \multicolumn{2}{|c|}{$\begin{array}{c}\text { Post Experiencia } \\
\text { Formativa }\end{array}$} & \multirow[t]{2}{*}{ Diferencia } \\
\hline & Media & Desv. St. & Media & Desv. St. & \\
\hline $\begin{array}{l}\text { Me gustaría utilizar las redes } \\
\text { sociales para las clases de } \\
\text { matemática }\end{array}$ & 2.24 & .822 & 2.70 & .953 & +0.46 \\
\hline $\begin{array}{l}\text { Las redes sociales se pueden } \\
\text { utilizar para resolver problemas } \\
\text { de matemática }\end{array}$ & 2.68 & .891 & 3.00 & .782 & +0.32 \\
\hline
\end{tabular}

Tabla 5. Medias, desviación y diferencia sobre pretest y postest de los aspectos relacionados con Matemática y resolución de problemas.

(en la utilización de las herramientas de comunicación de las RS, y el uso de las RS para la enseñanza de las matemáticas), antes y después de la experiencia formativa con un riesgo alfa de equivocarnos de $0.05 \%$. Lo que nos permite concluir que la experiencia formativa basada en redes sociales no ha influido en el uso que los alumnos le dan a la misma.

H1 (Hipótesis Alternativa): Existen diferencias significativas en el uso que realizan de las RS (en la utilización de las herramientas de comunicación de las RS, y el uso de las RS para la enseñanza de las matemáticas), antes y después de la experiencia formativa con un riesgo alfa de equivocarnos de $0.05 \%$. Lo que nos permite concluir que la experiencia formativa basada en redes sociales no ha influido en el uso que los alumnos le dan a la misma.

El estadístico que utilizaremos para ello fue el de Wilcoxon (Siegel, 1976) y en la Tabla 6 presentamos los resultados alcanzados.

Los valores alcanzados con el estadístico, nos permiten rechazar las tres hipótesis nulas formuladas y en consecuencia aceptar que la experiencia de utilización de la RS Edmodo para la resolución de problemas matemáticos había tenido consecuencias significativas para modificar las percepciones que los alumnos tenían inicialmente respecto a los usos que los estudiantes hacían de las RS, y de sus percepciones para ser utilizadas en las clases de matemáticas y para resolver problemas matemáticas. En todos los casos

\begin{tabular}{|l|c|c|c|c|}
\hline & Uso de las RS & $\begin{array}{c}\text { Uso de las } \\
\text { herramientas } \\
\text { delas RS }\end{array}$ & $\begin{array}{c}\text { Uso de las RS en } \\
\text { la clase de } \\
\text { matemáticas }\end{array}$ & $\begin{array}{c}\text { Uso de las RS } \\
\text { para resolver } \\
\text { problemas de } \\
\text { matemáticas }\end{array}$ \\
\hline & Postest-Pretest & Postest-Pretest & Postest-Pretest & Postest-Pretest \\
\hline $\mathbf{Z}$ & $-4,256$ & $-4,671$ & $-3,100$ & $-3,358$ \\
\hline Asymp. Sig. & $0.000(* *)$ & $0.000(* *)$ & $0.002(* *)$ & $0.001(* *)$ \\
\hline
\end{tabular}

Tabla 6. Test estadístico de Wilcoxon para las puntuaciones pretes-postest $\left(^{* *}=\right.$ significativo al nivel de significación del 0,01). 


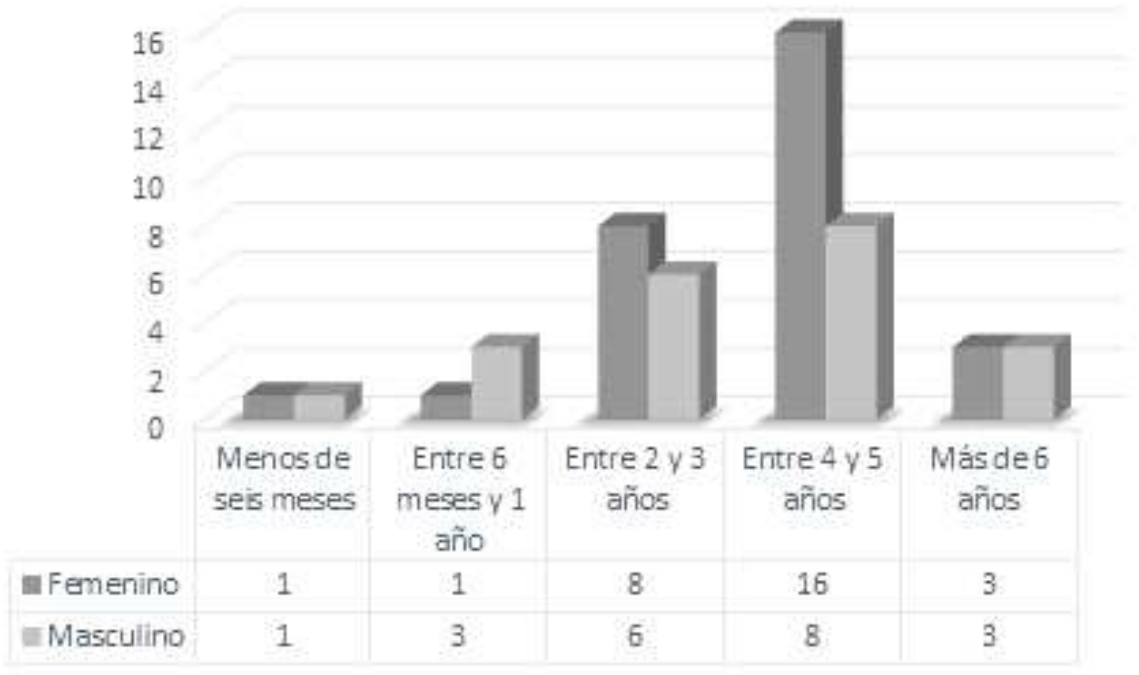

Figura 2. Relación entre el tiempo que tienen en la red y el género.

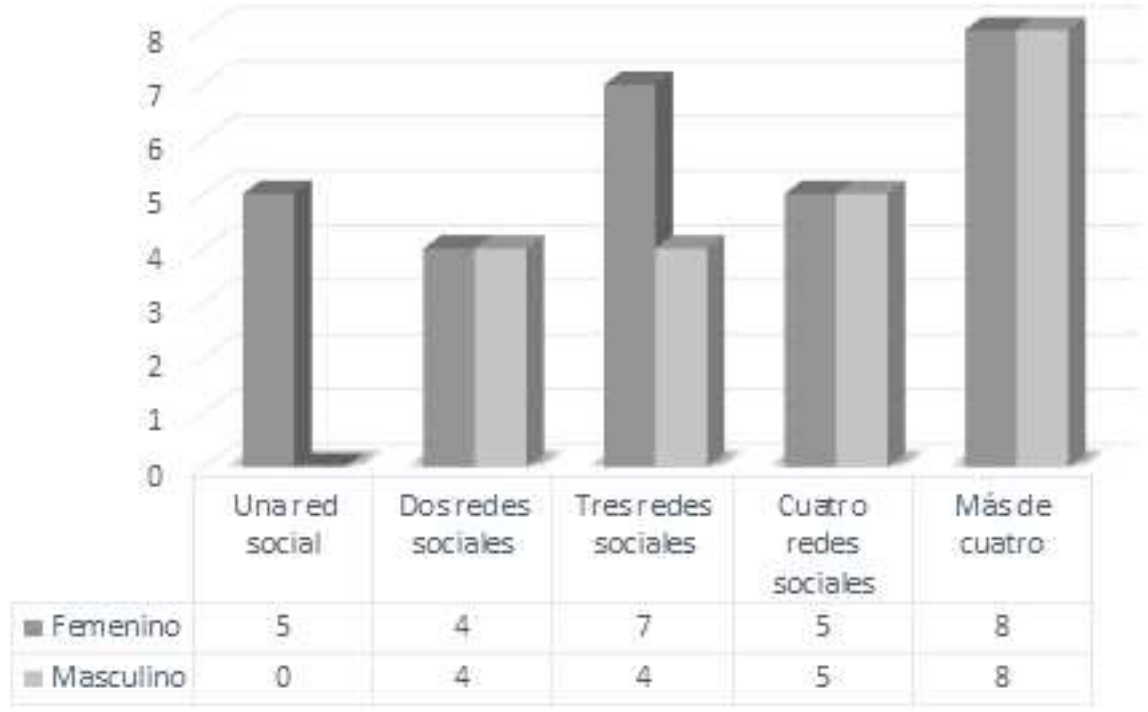

Figura 3. Relación entre la cantidad de perfiles en la red y el género. 


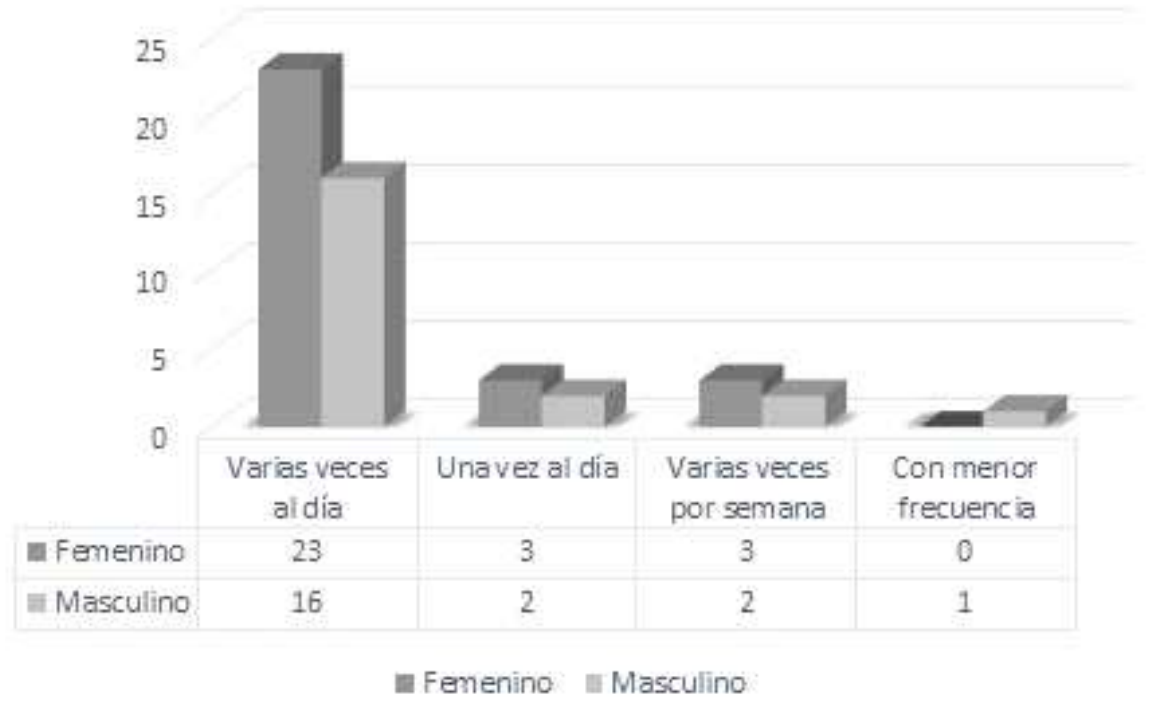

Figura 4. Relación tiempo que pasan en la red y el género.

las puntuaciones posteriores fueron superiores a las iniciales.

Uno de nuestros objetivos perseguía conocer si había diferencia en las contestaciones que los alumnos habían ofrecido en función del género. $\mathrm{Y}$ al respecto uno de nuestros primeros análisis buscó saber quiénes llevaban más tiempo con una cuenta activa en las RS y cómo podemos observar en la Figura 2, las chicas dominicana encuestadas invierten más tiempo en las redes que los chicos. En cierta medida podríamos decir que ellas siendo menores de 13 años ya tenían cuentas activas en la red.

También podemos observar en la Figura 3, el $55 \%$ ( $\mathrm{f}=16$ ) de las chicas han introducido su perfil en más de una red social y el $100 \%$ $(\mathrm{f}=21)$ tienen su perfil en más de una red. Obteniéndose resultados similares entre hombres y mujeres en las opciones de respuestas cuatro y más de cuatro RS.
Por lo que se refiere a quién pasaba más tiempo dentro de la red, podemos observar en la Figura 4 que las alumnas pasan más tiempo en las redes, en concreto el $79 \%$ ( $\mathrm{f}=23$ ) de ellas entran a sus cuentas varias veces al día. Lo que nos permite suponer que son más activas que los chicos.

En la Figura 5 podemos ver más claramente estas diferencias entre chicos y chicas, a través del análisis de los usos que hacen de ella, donde las chicas tienen las medias más altas de 3.76 en el uso de las redes sociales para mantener contacto con sus amigos y la más baja en conocer gente nueva, y los varones al igual que las chicas tienen la media más alta de 3.38 en mantener el contacto con sus amigos y la más baja de 2.33 en comentar.

Por lo que se refiere al uso que hacen de las RS en su tiempo en la red, podemos observar en la Figura 6, que ambos casos tanto chicas como chicos tienen la media más alta en hacer tareas y/o actividades del colegio 


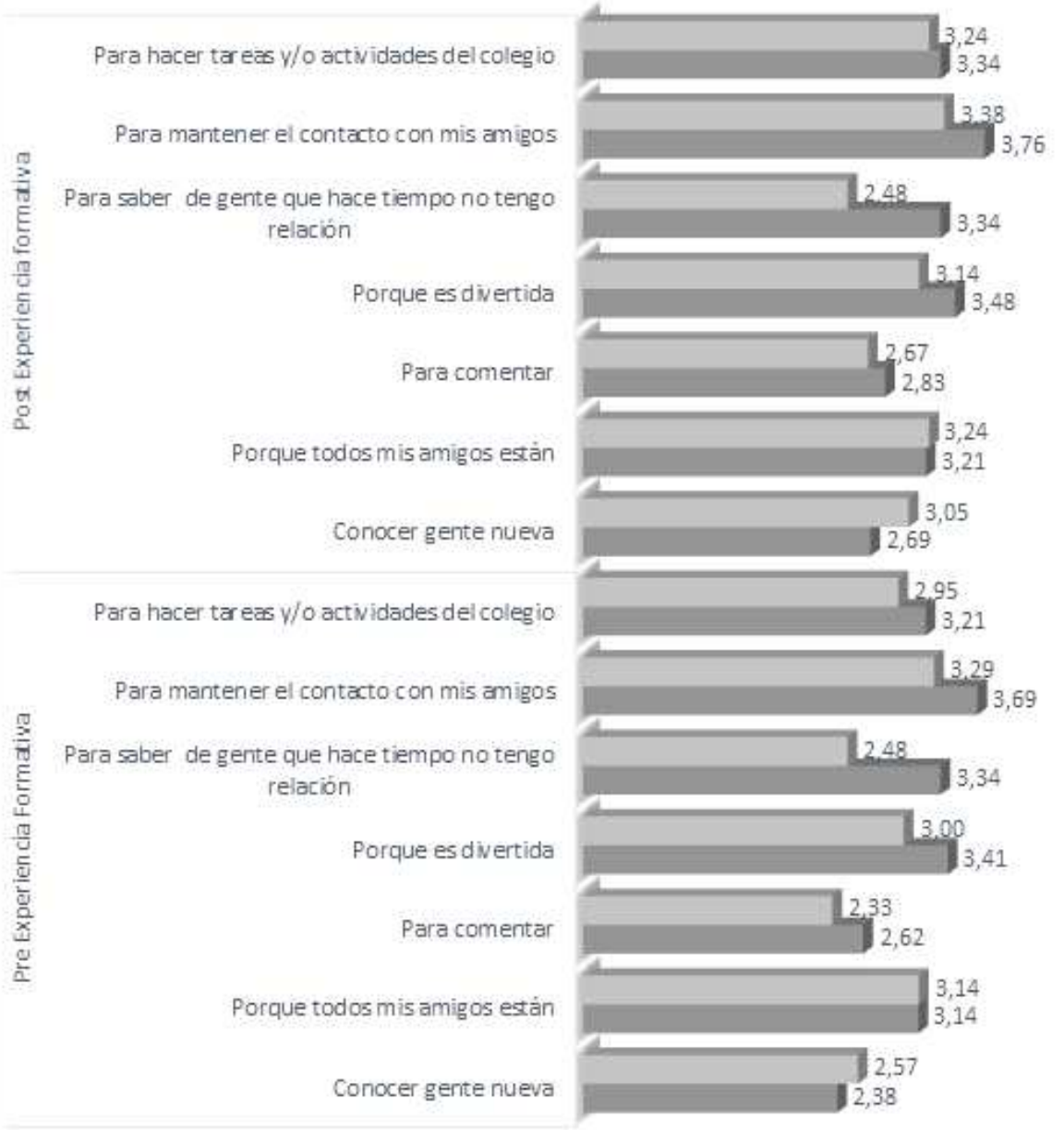

$0,00 \quad 0,501,001,502,002,503,003,504,00$

\section{Masculino = Femenino}

Figura 5. Relación entre uso de las redes sociales y género.

con 3.34 y 3.24 respectivamente y las más bajas de las chicas se encuentra en jugar en la red con una media de 1.62 y mandar mensajes privados con una media de 1.48. Al igual que las chicas los varones tienen las medias más bajas en las mismas herramientas con medias de 1.62 en mandar mensajes privados y 2.19 para jugar en la red.

Como podemos observar en la Figura 7 , antes de la experiencia formativa tanto los 


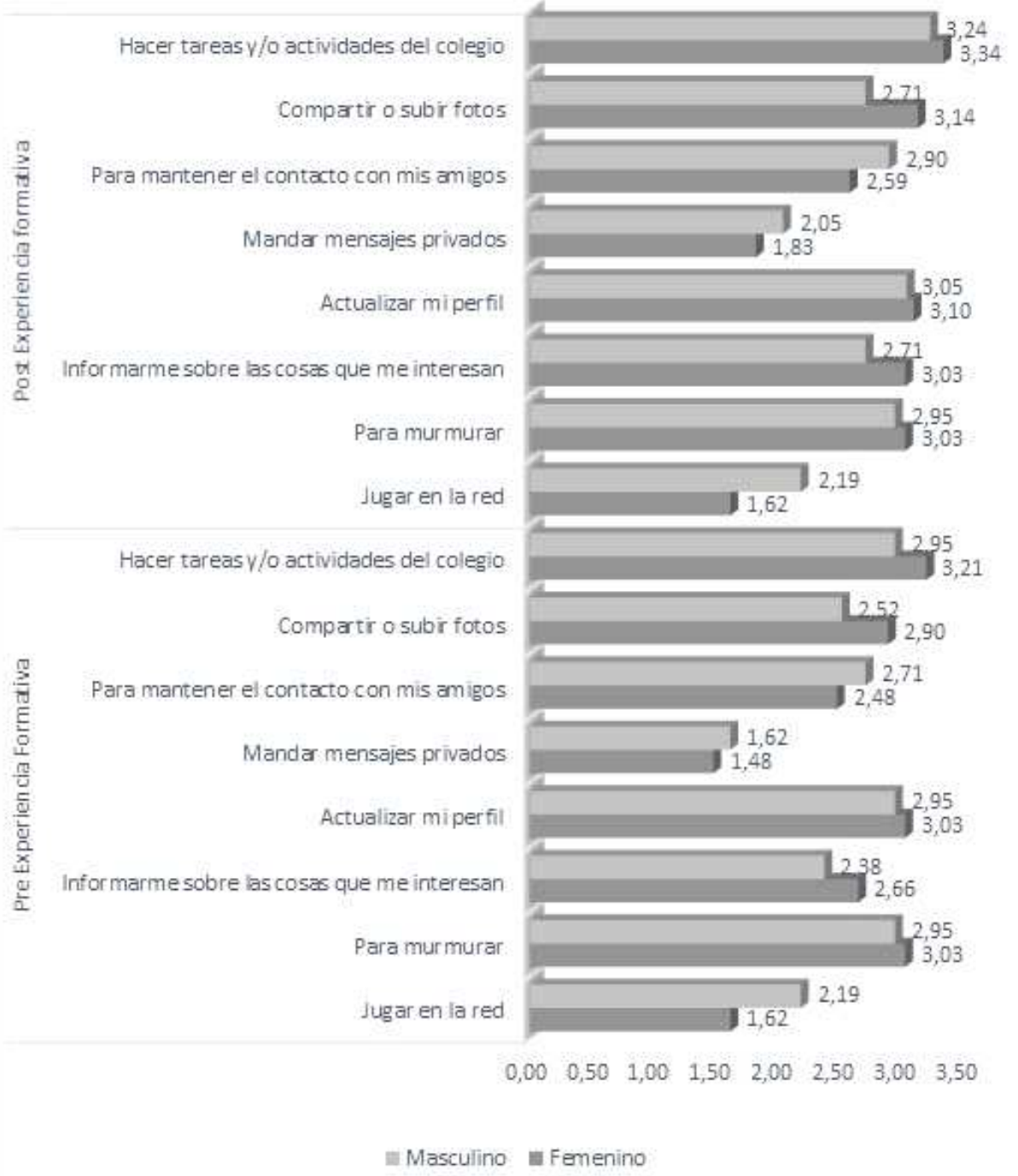

Figura 6. Medias de la uso de la herramienta de las redes sociales y género.

chicos como las chicas estaban en luego de la experiencia ambos están de desacuerdo con utilizar las redes sociales para resolver problemas matemáticos pero los chicos estaban más abiertos a utilizarla para la enseñanza de las Matemáticas, ahora bien, acuerdo con las dos afirmaciones aunque las chicas mantienen un poco de reserva para su uso en la resolución de problemas.

Píxel-Bit. Revista de Medios y Educación. No 48 Enero 2016. ISSN: 1133-8482. e-ISSN: 2171-7966. doi: http://dx.doi.org/10.12795/pixelbit.2016.i48.11 

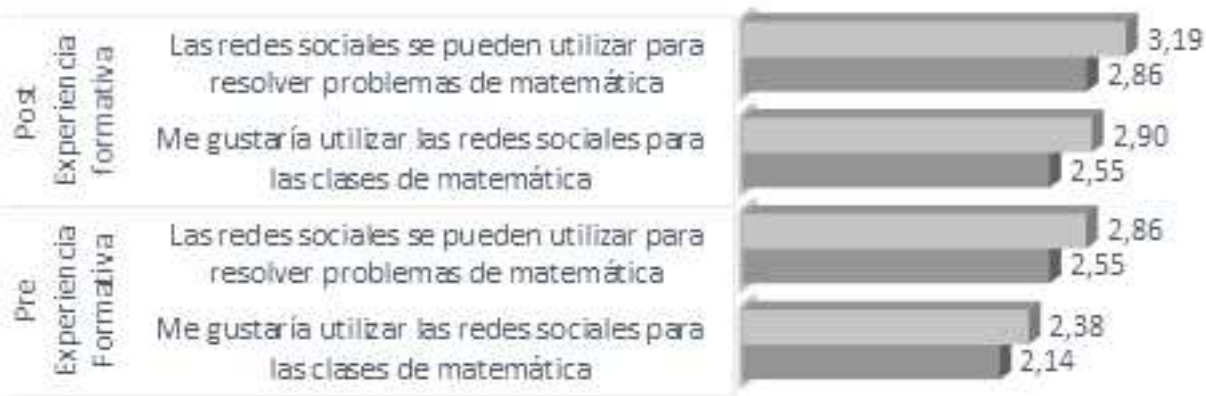

$0,000,501,001,502,002,503,003,50$

\section{Masculino a Femenino}

Figura 7. Medias del uso de las redes sociales para la enseñanza de las Matemáticas y la resolución de problemas y género.

\begin{tabular}{|l|r|r|r|r|r|r|}
\hline & \multicolumn{2}{|c|}{ Uso de las RS } & \multicolumn{2}{c|}{ Tiempo de uso } & \multicolumn{2}{c|}{ Enseñanza mat } \\
\hline & \multicolumn{1}{|c|}{ Pretest } & \multicolumn{1}{|c|}{ Posttest } & \multicolumn{1}{|c|}{ Pretest } & Posttest & Pretest & \multicolumn{1}{|c|}{ Posttest } \\
\hline Mann-Whitney U & 187.000 & 185.500 & 285.500 & 282.500 & 234.000 & 225.000 \\
\hline Wilcoxon W & 418.000 & 416.500 & 720.500 & 717.500 & 669.000 & 660.000 \\
\hline Z & -2.320 & -2.352 & -.375 & -.435 & -1.429 & -1.596 \\
\hline Asymp. Sig. (2-tailed) & .020 & .019 & .707 & .664 & .153 & .110 \\
\hline
\end{tabular}

Tabla 7. Valores U de Mann-Whitney en función del género.

Uno de nuestros objetivos era conocer si había habido diferencias significativas en las puntuaciones alcanzadas en las diferentes dimensiones antes y después en función del género del estudiante, y para ello formulamos las siguientes hipótesis:

H0 (Hipótesis Nula): No hay diferencias significativas en la frecuencia de utilización (el uso que hacen de las RS y las preferencias para utilizarlas en las clases de matemáticas), y el género de los estudiantes, con un riesgo alfa de equivocarnos de $0.05 \%$. Lo que nos permite concluir que el género del estudiante no tiene una influencia.
H1 (Hipótesis Alternativa): Existen diferencias significativas en la frecuencia de utilización (el uso que hacen de las RS y las preferencias para utilizarlas en las clases de matemáticas), y el género de los estudiantes, con un riesgo alfa de equivocarnos de $0.05 \%$. Lo que nos permite concluir que el género del estudiante no tiene una influencia.

El estadístico que utilizaremos para ello fue la U de Mann-Whitney (Siegel, 1976). Los resultados alcanzados los presentamos en la Tabla 7.

Como podemos observar solamente rechazamos las Hipótesis nulas formuladas 
respecto al tipo de uso que hacían de las RS, donde sí existieron diferencias entre las alumnas y los alumnos. Y ello tanto en las puntuaciones obtenidas antes de comenzar la experiencia y una vez finalizada la misma.

\section{Conclusiones.}

Nuestro trabajo nos permite señalar una serie de conclusiones:

Los estudiantes poseen cuentas activas en diferentes redes sociales, lo que se percibe tanto en las chicas como en los chicos.

- Las Chicas pasan más tiempo en las redes que los chicos y este tiempo lo utilizan para mantener contacto con sus amigos.

Pudimos observar que existen diferencias significativas entre los chicos y las chicas con respecto a la utilidad de las redes sociales y no así en el uso de sus herramientas ni en el uso de estás para las Matemáticas y la resolución de problemas.

No pudimos observar diferencias significativas entre las de edades de los alumnos y las etapas de experiencia formativa.

Observamos que hubo cambios significativos en ambas etapas de la experiencia formativa con respecto al uso, herramientas y utilidad hacia las Matemáticas.

Se observan lo cambiante de las redes y los jóvenes ya que entre el tiempo que se aplica el Pre-test y el Post-test los estudiantes se dan de alta en nuevas redes sociales.

Las redes más utilizadas por los jóvenes son Facebook, Twitter, Youtube e Instagram esta última de preferencia de las chicas más que de los chicos.

Las chicas prefieren redes donde puedan postear fotos y mini videos no así los chicos que prefieren redes donde puedan ver videos.

Los estudiantes tienen una actitud positiva ante el uso de las redes sociales para la enseñanza de las Matemáticas y la resolución de problemas.

\section{Referencias bibliográficas.}

Arnold, N. \& Trena, P. (2010). Using a social networking site for experiential learning: Appropiating, lurking, modeling and community building. Internet and Higher Education, 13(4), 188-196. doi:10.1016/ j.iheduc. 2010.04.002

Aydin, S. (2012). A review of research on Facebook as an educational environment. Education Tech Research Development, 60(6), 1093-1106. doi: 10.1007/s11423-0129260-7

Barroso, J. \& Cabero, J. (2010). La investigación en TIC. Madrid: Sintesis.

Boyd, D. \& Ellison, N. (2007). Social Network Sites: Definition, History and Scholarship. Journal of computer-mediated communication, 13(1), 210-230. Recuperado el 2013 de Enero de 2013, de http:// onlinelibrary.wiley.com/doi/10.1111/j.10836101.2007.00393.x/epdf. doi:10.1111/j.10836101.2007.00393.x

Cabero, J. (2012). Las redes sociales en el entramado educativo de la Web. 2.0. En E. Navas, Web 2.0 Innovación e investigación educativa (pp. 11-28). Caracas: Univ. Metropolitana.

Cabero, J., Barroso, J., Llorente, M. \& Marín, V. (2013). Las Redes Sociales como instrumento para la formación: Percepciones de los alumnos universitarios hacia el trabajo en grupo. Sevilla: Grupo de Investigación Didáctica:Universidad de Sevilla. 
Cabero, J. \& Marín, V. (2013). Percepciones de los estudiantes universitarios latinoamericanos sobre las redes sociales y el trabajo en grupo. RUSC. Universities and Knowledge Society Journal, 10(2), 219-235. doi: <http://doi.dx.org/10.7238/ rusc.v10i2.1728>

Cachia, R. (2008). Los sitios de creación de redes. Aspectos Sociales. Revista Telos, 76. Recuperado el 22 de Enero de 2013, de http:/ /telos.fundaciontelefonica.com/telos/ articulocuaderno.asp@idarticulo=4\&rev=76.htm

Camacho, M. (2010). Las redes sociales para enseñar y aprender. Reflexiones pedagógicas básicas. En L. Castañeda, Aprendizajes con redes sociales. Tejidos educativos para los nuevos entornos (pp.91104). Sevilla: Eduforma.

Chávez, M., Solis, N. \& Iriarte, A. (2012). Estrategias de Aprendizaje Colaborativo para Redes Sociales Digitales. XX Encuentro Internacional de Educación a Distancia: Redes y alianza más allá de las modalidades educativas. Jalisco, México: UDG Virtual.

De Alsola, J. (2009). Las Redes Sociales satisfacen necesidades básicas. Recuperado el 15 de enero de 2013, de slideshare: http:// www.slideshare.net/Julianalsola/las-redessociales-1649666

De Haro, J. (2010). Redes Sociales para la educación. Madrid: Anaya.

De Haro, J. (2012). Servicio de redes sociales para la educación. Recuperado el 14 de noviembre de 2013, de Educativa: http:/ /jjdeharo.blogspot.com/2012/03/servicios-deredes-sociales-para-la.html

Durall, E., Gros, B., Maina, M., Johnson, L. \& Adams, S. (2012). Perspectivas tecnológicas: educación superior en Iberoamérica 2012-2017. Austin, Texas: The New Media Consortium.
García, I., Peña, I., Johnson, L., Smith, R., Levine, A. \& Haywood, K. (2010). Informe Horizon: Edición Iberoamericana 2010. Austin, Texas: The new Media Consortium.

Irwin, C., Ball, L., Desbrow, B. \& Leveritt, M. (2012). Students' perceptions of using Facebook as an interactive learning resource at university. Australasian Journal of Educational Technology, 28(7), 1221-1232.

Joosten, T. (2012). Social Media for Educators. Strategies and best practices. California: Jossey-Bass.

Llorente, M. (2012). Educar a través de la red. Evolución y aspectos educativos a tener en cuenta. En E. Navas, Web 2.0 Innovación e investigación educativa (pp. 93-115). Caracas: Univ. Matropolitana.

López, E., Vásquez, E. \& Sarasola, J. L. (2015). Estudio bibliométrico de Pixel-bit, revista de medios y educación (2000-2013). Pixel-Bit. Revista de Medios y Educación, 46, 65-85. doi: http://dx.doi.org/10.12795/ pixelbit.2015.i46.05

McMillan, J. H. \& Schumacher, S. (2005). Investigación educativa: una introducción conceptual. Madrid: Pearson.

Minocha, S., Schroeder, A. \& Schneider, C. (2011). Role of the educator in social software initiatives in further and higher education: A conceptualization and research agenda. British Journal of Educational Technology, 42(6), 889-903. doi: 10.1111/j.14678535.2010.01131.x

Ortega, S. \& Gacitúa, J. (2008). Espacios interactivos de comunicación y aprendizaje. La construcción de identidades. Revista de Universidad y Sociedad del Conocimiento (RUSC), 5(2), 17-25. Recuperado el 14 de Enero de 2013, de http://www.uoc.edu/rusc/ 5/2/dt/esp/ortega_gacitua.pdf

Osborne, C. (2012). The pros and cons of social media classrooms. Recuperado el 10 
de febrero de 2013, de ZDnet: http:// www.zdnet.com/blog/igeneration/the-prosand-cons-of-social-media-classrooms/15132

Rodríguez, R. E. (2013). Las redes sociales como estrategia para lograr un aprendizaje significativo de la Historia de México. Revista Congreso Universidad, 2(1). Recuperado de http://www.congresouniversidad.cu/revista/ index.php/congresouniversidad/article/ viewFile/245/230

Roig, R. (2011). Redes Sociales educativas. Propuestas para la intervención en el aula. En F. Matínez y I. Solano, Comunicación y relaciones sociales de los jóvenes en la red (pp. 137-151). Murcia: Marfil.

Romero, J. (2011). Las redes sociales en educación. Revista digital CIS-F. Recuperado el 24 de enero de 2014, de http://www.csi-f.es/ archivos/andalucia/ensenanza/revistas/iee/ $\begin{array}{llllllllll}\mathrm{N} & \mathrm{u} & \mathrm{m} & \mathrm{e} & \mathrm{r} & \mathrm{o} & & 4 & 4 & \text { / }\end{array}$ JOSE_LUIS_ROMERO_LACAL_1.pdf

Sampieri, R., Fernández, C. \& Baptista, P. (2013). Metodología de la Investigación (5ta. Ed. ed.). México D. F: Mc Graw-Hill.

Santamaría, F. (2008). Posibilidades pedagógicas. Redes sociales y comunidades educativas. TELOS. Cuaderno de comunicación e innovación, 76. Recuperado de http://telos.fundaciontelefonica.com/ $\begin{array}{llllll}\mathrm{t} & \mathrm{e} & \mathrm{l} & \mathrm{o} & \mathrm{s} & /\end{array}$ articulocuaderno.asp@idarticulo=7\&rev=76.htm

Siegel, S. (1976). Estadística no paramétrica. México: Trillas.

Túñez, M. \& Sixto, J. (2012). Las redes sociales como entorno docente: análisis del uso de Facebook en la docencia Universitaria. Pixel-Bit. Revistas de Medios y Educación, 41,77-92.

Veletsianos, G. \& Navarrete, C. (2012). Online social networks as formal learning environments: Learner experiences and activities. The International Review of
Research in Open and Distributed Learning, 13(1), 144-166.

Fecha de recepción: 01-09-2015

Fecha de evaluación: 14-10-2015

Fecha de aceptación: 21-10-2015 


\section{Anexo.}

Cuestionario Percepción hacia las redes sociales.

El siguiente cuestionario no tiene ninguna nota, no hay respuestas correctas ni incorrectas, y por lo tanto no tiene consecuencia en sus calificaciones. Solo deseamos saber si usted está de acuerdo o en desacuerdo con cada una de las siguientes afirmaciones

Muchas Gracias por tu cooperación

\begin{tabular}{|c|c|c|c|c|c|}
\hline \multicolumn{6}{|c|}{ Datos Generales: } \\
\hline 1. & Género: & 2. & Edad: & 3. & Grado: \\
\hline$\square$ & Femenino & $\square$ & 13 años & $\square$ & 1 de Media \\
\hline \multirow[t]{5}{*}{$\square$} & Masculino & $\square$ & 14 años & $\square$ & 2 de Media \\
\hline & & $\square$ & 15 años & $\square$ & 3 de Media \\
\hline & & $\square$ & 16 años & $\square$ & 4 de Media \\
\hline & & $\square$ & 17 años & & \\
\hline & & $\square$ & Más de 17 años & & \\
\hline
\end{tabular}

\section{Instrucciones:}

No tome mucho tiempo en ninguna de las afirmaciones, más bien asegúrese de responder a cada una de ellas. Trabaje con cuidado. Recuerde que no hay respuestas correctas o incorrectas, lo que interesa es su opinión. Deje que su experiencia lo guíe para marcar su verdadera opinión. 


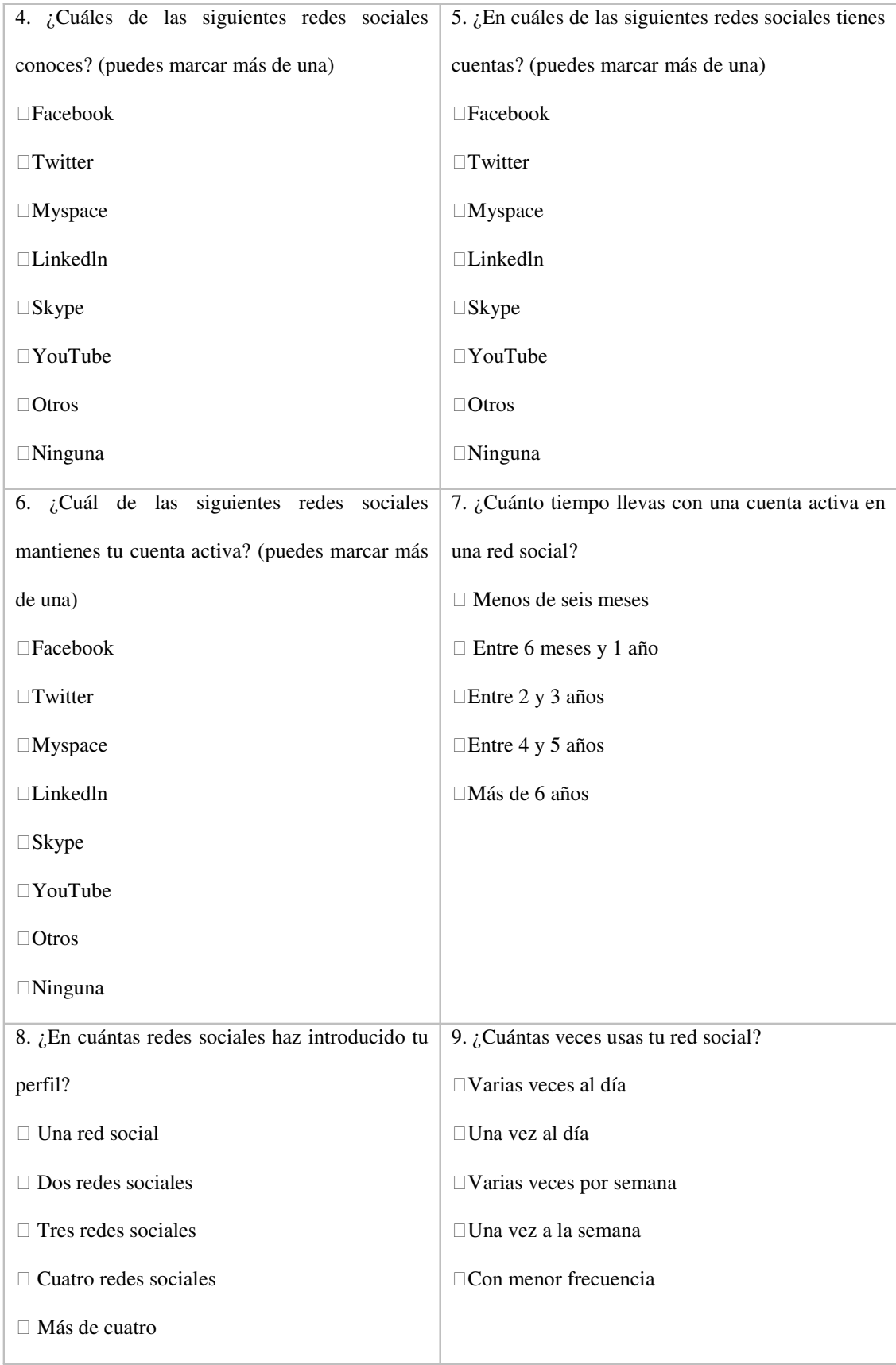




\begin{tabular}{|c|c|c|c|c|}
\hline & 1 & 2 & 3 & 4 \\
\hline Utilizo las redes sociales para: & & & & \\
\hline 10. Conocer gente nueva & & & & \\
\hline 11. Porque todos mis amigos están & & & & \\
\hline 12. Para comentar & & & & \\
\hline 13. Porque es divertida & & & & \\
\hline 14. Para saber de gente que hace tiempo no tengo relación & & & & \\
\hline 15. Para mantener el contacto con mis amigos & & & & \\
\hline 16. Para hacer tareas y/o actividades del colegio & & & & \\
\hline Utilizo mí tiempo en la red social para: & & & & \\
\hline 17. Jugar en la red & & & & \\
\hline 18. Para murmurar & & & & \\
\hline 19. Informarme sobre las cosas que me interesan & & & & \\
\hline 20. Actualizar mi perfil & & & & \\
\hline 21. Mandar mensajes privados & & & & \\
\hline 22. Comentar las fotos de mis amigos & & & & \\
\hline 23. Compartir o subir fotos & & & & \\
\hline 24. Hacer tareas y/o actividades del colegio & & & & \\
\hline 25. Me gustaría utilizar las redes sociales para las clases de matemática & & & & \\
\hline 26. Las redes sociales se pueden utilizar para resolver problemas de matemática & & & & \\
\hline
\end{tabular}

(Nota: 1=totalmente en desacuerdo, 2=en desacuerdo, 3=de acuerdo, 4=totalmente de 